Running head: Blink adaptation for target displacements in depth.

Blink adaptation for target displacements in depth

Arnab Biswas, Gerrit W. Maus

Psychology, School of Social Sciences

Nanyang Technological University

Singapore 


\begin{abstract}
To maintain gaze stability, the oculomotor system needs to correct gaze position errors that may emerge subsequent to an eye blink. It has been shown that artificially induced gaze position errors, when a target is displaced by a consistent amount during an eye blink, lead to a recalibration of gaze control during the blink, a process termed 'blink adaptation' (Lau \& Maus, 2018; Maus et al., 2017). In the present study, we investigate if this corrective mechanism also occurs for errors induced by displacing the target in depth during an eye blink. Across two conditions, subjects were asked to fixate on a target, whose disparity was increased or decreased when the eyelids were completely closed during a blink. We found that participants recalibrated their gaze for both conditions, with more automatic vergence eye movements occurring during the blink in the direction required to correct for the disjunctive step of the target. This corrective effect was generally larger for the condition with decreasing stimulus disparity as compared to increasing disparity. Our results reveal that blink adaptation is a general recalibration mechanism in oculomotor control, which can be driven by both monocular errors that correct for conjugate gaze targeting errors, or by binocular errors that correct for disconjugate vergence errors.
\end{abstract}

Keywords- blinks, vergence, adaptation, eye-movements 


\section{BLINK ADAPTATION FOR TARGET DISPLACEMENTS IN DEPTH}

\section{Introduction}

In humans, visual resolution decreases dramatically as we move away from the fovea, the center of our vision, to the periphery (Green, 1970). Thus to maintain visual acuity, the oculomotor system needs to continually move our eyes to foveate onto areas of interest around us. It does so by directing our eyes to make coordinated saccades, smooth pursuits, and vergence eye movements.

The classical proposition made by Hering (1868) notably identified two distinct subsystems that are involved in eye movement control. A conjugate change in the same direction for both eyes (version) is attributed to a saccadic subsystem. On the other hand, a separate vergence subsystem controls disjunctive shifts in the two eyes, usually to compensate for a change in depth. Many studies have experimentally confirmed this dichotomy based on characteristic differences in vergence and version dynamics, such as a difference in peak velocities (Westheimer, 1954; Rashbass \& Westheimer, 1961), or based on neurophysiological differences observed in primate studies (Mays, 1984; Mays, Porter, Gamlin \& Tello, 1986; Gamlin, 2002).

Eye-movements are motor processes which can be noisy and error-prone. Furthermore, through the course of life, the change in interpupillary distance, loss of conjugacy after spectacle correction, or displacement of extraocular muscles due to aging, can all introduce consistent errors in gaze control, which can add up if not corrected. Thus like every robust control system, the oculomotor system also needs to recalibrate itself in response to pathological, physiological, and anatomical changes to maintain precise gaze control. Multiple studies have investigated adaptation for both the saccadic and vergence subsystems in response to induced 


\section{BLINK ADAPTATION FOR TARGET DISPLACEMENTS IN DEPTH}

errors. Saccadic plasticity is well documented using the intra-saccade step paradigm (for reviews, see Hopp \& Fuchs, 2004; Iwamoto \& Kaku, 2010; Pélisson, Alahyane, Panouillères, \& Tilikete, 2010). Other studies have shown an indication of disconjugate saccadic adaptation by introducing a dichoptic step at the end of a saccade (Albano \& Marrero, 1995; Maiello, Harrison \& Bex, 2016, Kosovicheva \& Bex, 2020).

The plasticity of vergence eye movements has also been demonstrated by using prisms to induce a retinal disparity. After wearing such prisms for a long time, the eye offsets the added retinal disparity by developing a tonic-vergence angle equal to the prism angle (Flohr \& Precht, 1981). Additionally, it has been shown that stereopsis can be restored in adults with amblyopia by exposing them to stereoscopic 3D video games (Li et al., 2018).

Eye-blinks are another common source of error in gaze position. Humans usually blink around 15 to 20 times per minute (Evinger, 1995). During each blink, the eyes move nasally and downwards and then temporally and upwards (Collewijn, Steen, \& Steinman, 1985; Riggs, Kelly, Manning, \& Moore 1987). Gaze position errors are introduced during these blink-related eye movements, which are corrected by microsaccades and torsional movements (Costela et al., 2014; Khazali, Pomper, \& Thier, 2017). Recent studies have shown that to offset consistent gaze targeting errors across blinks, we recalibrate our gaze using an adaptive process termed "blink adaptation" (Maus et al., 2017; Lau \& Maus 2019). Maus et al. (2017) asked participants to fixate on a dot that was displaced to the right by a consistent amount whenever the participants blinked. Displacing the dot while the participants' eye lids were closed induced a conjugate gaze targeting error in the tracked eye just after the 


\section{BLINK ADAPTATION FOR TARGET DISPLACEMENTS IN DEPTH}

blink. It was observed that repeatedly inducing such errors resulted in the gradual adaptation of the post-blink gaze position towards the direction of the dot displacement, even while subjects were unaware of it. That is, with time the oculomotor system learned to anticipate the gaze targeting error and compensated for it by making larger eye movements in the direction of the dot displacement. Expanding upon this, in another study, Lau \& Maus (2019) showed that blink adaptation corrects for conjugate errors introduced by target displacements in the four cardinal directions, and that blink adaptation takes place for both voluntary and reflexive eye-blinks.

Building on these two studies, in this experiment we investigate if blink adaptation also occurs for disconjugate errors in gaze, induced by target displacements in depth during an eye blink. Subjects were asked to voluntarily blink while fixating on a dot. During each blink, the retinal disparity of the dot was either increased or decreased by a consistent amount, across separate conditions. For the duration of the experiment, we recorded the participant's binocular gaze position. If there is blink adaptation, we expect to find a positive change in the vergence angle across blinks for the increasing disparity condition and a negative change in vergence for the decreasing disparity condition.

\section{Methods}

\section{Participants}

Thirteen participants were recruited from the Nanyang Technological University research participant pool. All of them received course credits for their time. Data from 


\section{BLINK ADAPTATION FOR TARGET DISPLACEMENTS IN DEPTH}

three of these participants were not used for analysis as we noticed that these participants had shifted their heads during the course of the experiment leading to loss of pupil signal to the eye tracker, which led to disruption in the control of the stimuli. The remaining ten participants (6 females, age $18-24$, mean age $=21.3$ ) had normal or corrected to normal vision. All procedures were approved by the Nanyang Technological University Institutional Review Board. All participants provided written informed consent.

\section{Apparatus and Stimuli}

We used the Psychtoolbox-3 (Brainard, 1997; Pelli, 1997; Kleiner, Brainard, \& Pelli, 2007) in MATLAB (MathWorks, Natick, MA) to program and present the stimuli. The stimuli were presented on a 20-inch Sun Microsystems CRT monitor with a refresh rate of $120 \mathrm{~Hz}$ and a resolution of $1280 \times 720$. Participants were seated in a dark room at a distance of $65 \mathrm{~cm}$ from the screen, with their heads stabilized using a chinrest. Participants wore active 3D shutter glasses (Nvidia 3D Vision wired glasses, Nvidia Corp., Santa Clara, CA) to control stereoscopic stimuli presentation. Horizontal eye position and pupil area was recorded binocularly at $1000 \mathrm{~Hz}$ using an Eyelink 1000+ (SR Research, Ottawa, Canada) video-based eye tracker. 


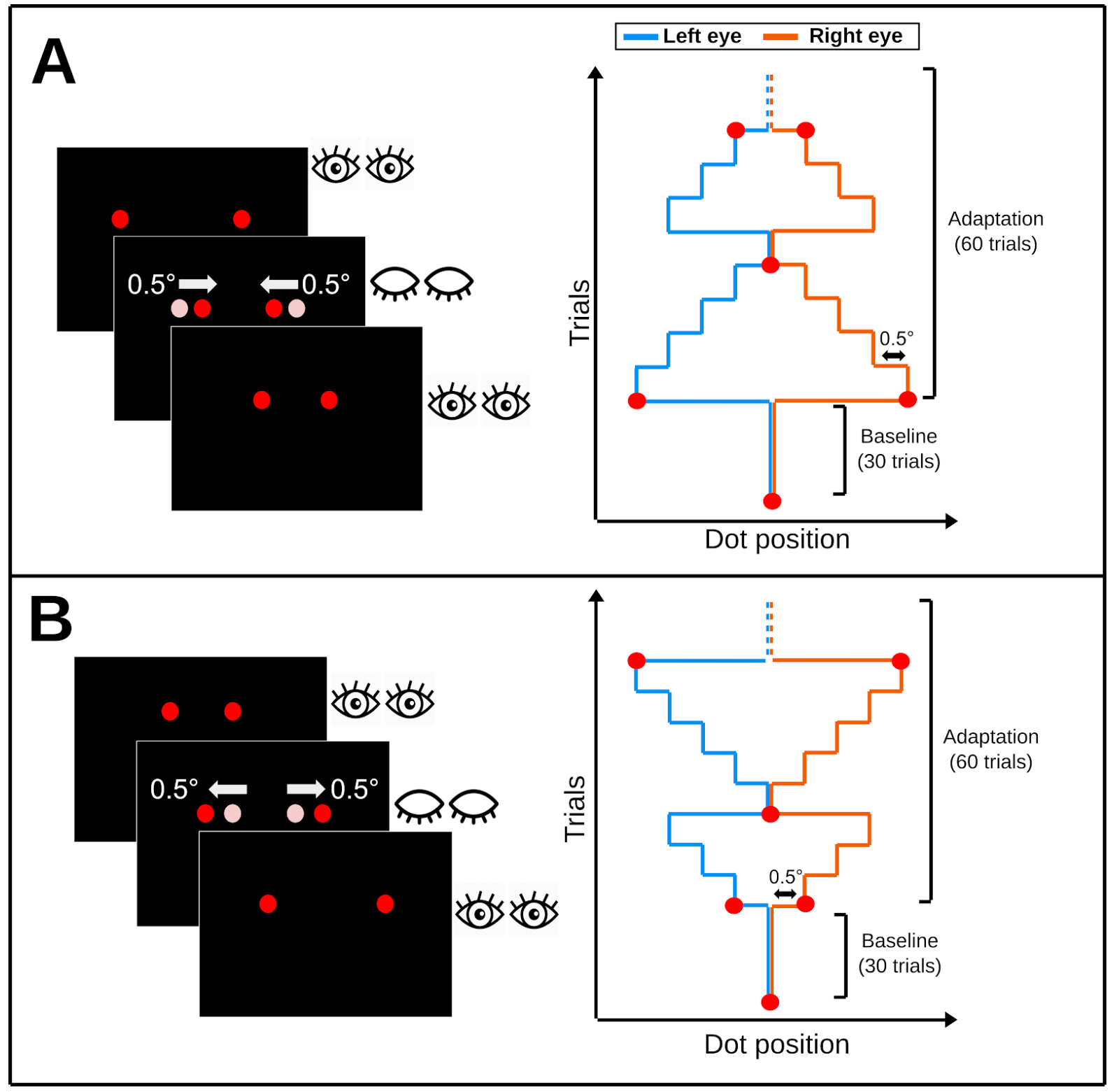

Figure 1: Participants were asked to fixate on a dot while their binocular gaze position was recorded using an eye tracker. During the adaptation phase, every time a blink was detected, the retinal disparity of the dot was either decreased (A) or increased $(B)$ depending on the condition. There was no change in disparity for the baseline condition.

The stimulus was a circular red dot (diameter $=0.2^{\circ}$, luminance $=25.4 \mathrm{~cd} / \mathrm{m}^{2}$ ) presented separately to each eye using 3D shutter glasses on a black background 


\section{BLINK ADAPTATION FOR TARGET DISPLACEMENTS IN DEPTH}

(Figure 1) $\left(\right.$ black $=0.75 \mathrm{~cd} / \mathrm{m}^{2}$, white $\left.=109 \mathrm{~cd} / \mathrm{m}^{2}\right)$. At the start of the experiment, the dot was displayed randomly within a region $4.5^{\circ} \times 4.5^{\circ}$ visual angle around the center of the screen. These initial stimulus positions were distributed symmetrically about the straight-ahead position (cyclopean axis) across subjects, to avoid asymmetries in vergence offset between eyes. During the adaptation phase, after the onset of a blink, at the moment of complete eyelid closure, the dots were consistently displaced either temporally or nasally, based on the condition, in each eye by $0.5^{\circ}$. This displacement resulted in a change in retinal disparity. When the dots were displaced temporally in each eye, the retinal disparity of the stimulus increased. For the other condition, where the pair of dots were displaced nasally in each eye after every blink, the retinal disparity was decreasing equivalent to a dot constantly moving closer to the subject after each blink. After every 2 to 6 blinks, the disparity between the dots was returned to the original value, from where the disparity was again increased or decreased as per the condition. This was done to avoid the stimulus disparity from crossing the participant's stereo thresholds.

\section{Procedure}

In the experiment, participants were instructed to fixate on the dot continuously and encouraged to blink. The participants were informed that over time the dot would move around randomly in 3D space but participants were unaware of the fact that the dot would be displaced during blinks. For the first 30 trials/blinks - termed as the baseline phase, the stimulus disparity was maintained at $0^{\circ}$. For the next 60 trials, referred to as the adaptation phase, the stimulus disparity was consistently increased or decreased during every blink depending on the experimental condition. 


\section{BLINK ADAPTATION FOR TARGET DISPLACEMENTS IN DEPTH}

Conditions were counterbalanced across participants. During the experiment, we measured the binocular gaze position of the subjects just before and after the blink, to determine the change in vergence across blinks.

\section{Analysis}

\section{Eye tracker data analysis}

The analysis procedure was similar to previous studies in blink adaptation (Lau \& Maus, 2019, Maus et al. 2017). The $60 \mathrm{~Hz}$ shuttering of the 3D shutter glasses introduced a consistent noise in the gaze position and pupil area signal. This was filtered out using a Savitzky Golay filter (Savitzky \& Golay, 1964) of order 1 and window size 17 .

For our experiment, we were interested in the gaze position just before and just after the blink. We analyzed only horizontal gaze positions. Eye blinks were detected online using pupil area data from the eye tracker. For our experiment, a blink was defined to start when the pupil was completely occluded by the eyelid (pupil area $=0$ ) and the blink was determined to have ended when the post-blink pupil area exceeded $80 \%$ of the pre-blink pupil area. The Eyelink $1000+$ video-based eye tracker uses the pupils to calculate the gaze position. Partial/complete occlusion of the pupils after the blink introduces errors in the gaze position. Hence, only a continuously stable and non zero pupil area was taken as an indicator for reliable gaze position unaffected by eye tracker artefacts. Gaze position after the blink was considered to be stable when the variance within a 10 ms sliding window was 
determined to be less than 1.5 times the variance of the gaze position in a $100 \mathrm{~ms}$ window 150 to $50 \mathrm{~ms}$ before the blink onset.

The pre-blink gaze position was calculated by averaging the gaze position, within a $100 \mathrm{~ms}$ window, 150 to $50 \mathrm{~ms}$ before the blink onset. While the post blink gaze position was determined by averaging the first $100 \mathrm{~ms}$ after the end of the blink, or the stable gaze position till before a saccade was initiated, whichever was shorter. To calculate the adapted gaze position we subtracted the post-blink gaze position from the pre-blink gaze position. Vergence was calculated as the difference in gaze

position between the right and left eye, Vergence $=R_{\text {gaze }}-L_{\text {gaze }}$. That is, if the right and left eye were looking at the same location on the screen, then the vergence would be zero. A positive change in vergence denotes a diverging eye movement while a negative change represents a converging eye movement. Contrarily, any conjugate change in eye movement does not cause a change in vergence.

\section{Statistical analysis}

Based on our hypothesis, if there was blink adaptation, we would expect an increase in the post-blink vergence over the time course of the experiment for the increasing disparity condition and a decrease in post-blink vergence for the decreasing disparity condition. To compare the change in post-blink vergence during the adaptation phase we divided the adaptation trials into two chunks, the first 30 trials for each subject was denoted as the early adaptation phase and the later 30 trials which followed, were called the late adaptation phase. We used a $2 \times 3$ repeated measures ANOVA to analyze if there were any differences in vergence for the two 


\section{BLINK ADAPTATION FOR TARGET DISPLACEMENTS IN DEPTH}

conditions across the three phases. In case of a significant main effect, we used Bonferroni adjusted pairwise t-tests to check their validity.

Gaze and pupil data were processed and plotted using MATLAB. All statistical analyses were performed in R and JASP. The R package sjstats was used to calculate the effect size.

\section{Results}

To test our hypothesis, we first plotted the post-blink vergence time course for both increasing and decreasing disparity averaged across participants for all the trials (see Figure 2). We observe that the post-blink vergence during the adaptation for both the conditions is different from the post-blink vergence observed during the baseline phase. The post-blink vergence becomes smaller across adaptation trials for the decreasing disparity condition and larger for the increasing disparity condition.

Next we plotted the mean post-blink vergence for each of the three phases of the experiment (baseline, early adaptation, and late adaptation) for both increasing and decreasing disparity (see Figure 3) and compared the means using a repeated-measures ANOVA. We observed an interaction between the phase and the condition $\left(F(2,18)=16.097, p<0.001, \eta^{2}=0.062\right)$. There were no significant main effects for phase $\left(F(2,18)=1.038, p=0.374, \eta^{2}=0.009\right)$ or condition $(F(1,9)=4.538$, $\left.p=0.062, \eta^{2}=0.091\right)$. 


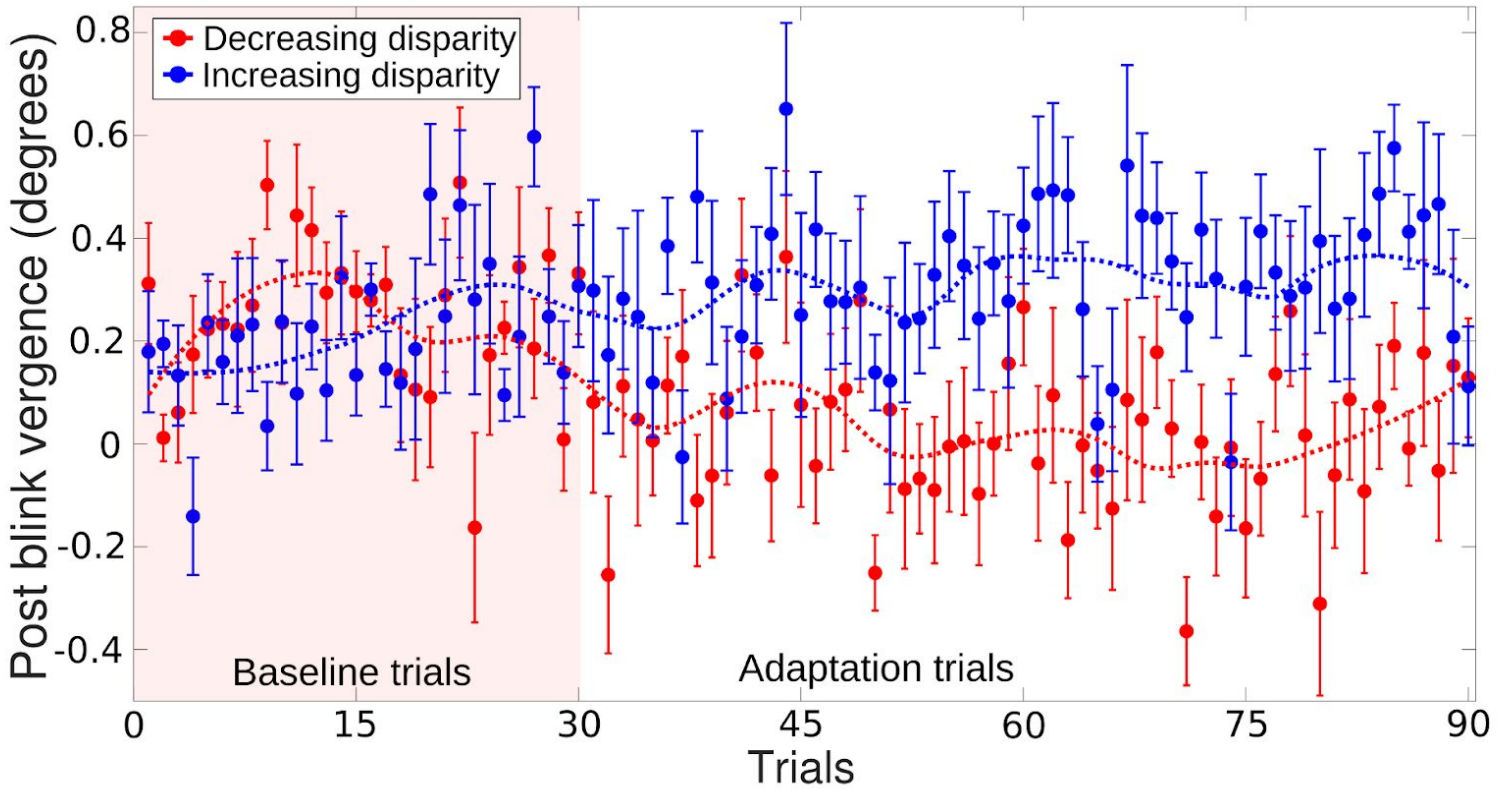

Figure 2: Mean Post-blink vergence across observers for both increasing and decreasing disparity conditions plotted for the time course of the experiment. The dotted lines represent a loess regression performed with smoothing factor=0.25. Error bars are standard errors of means. The post-blink vergence for the decreasing disparity condition (red) shows a decreasing trend and a clear separation from the increasing disparity. 


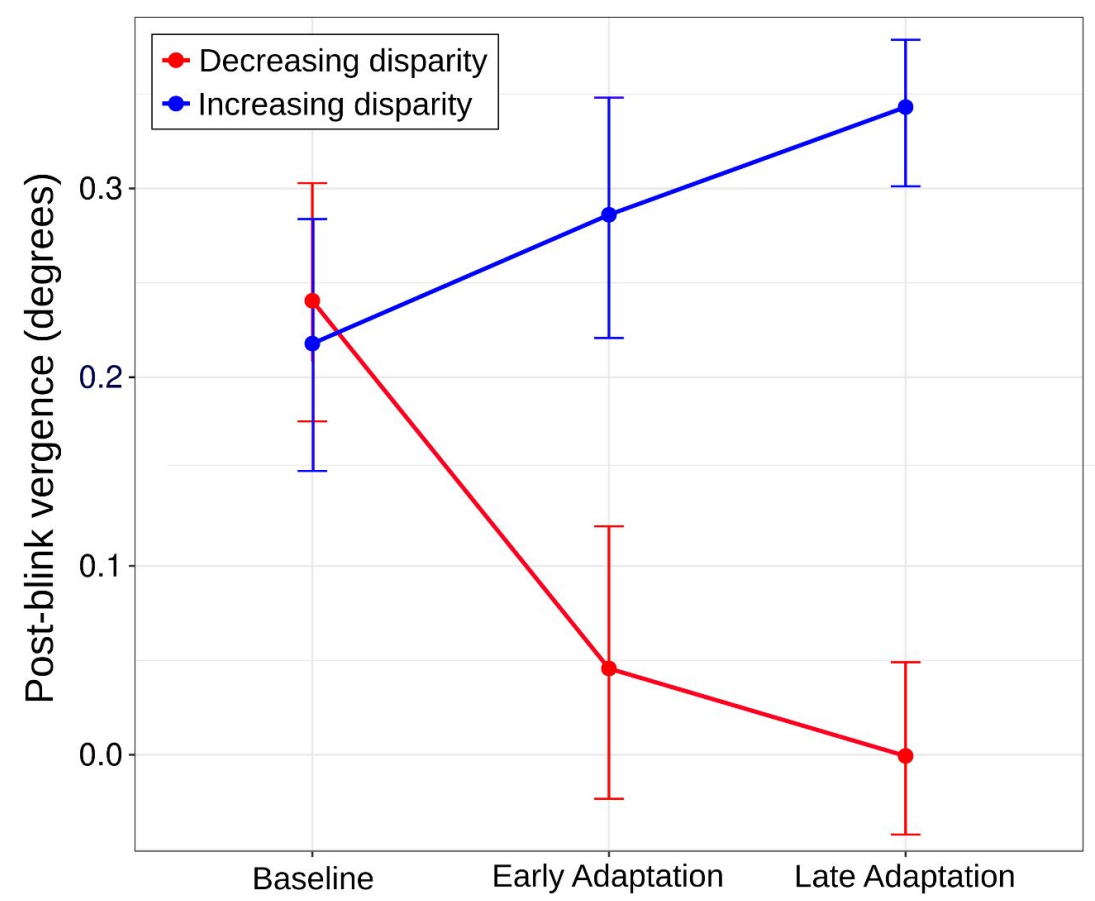

Figure 3: Post-blink vergence for both increasing and decreasing disparity conditions grouped by the different phases (Baseline trials 1-30, Early adaptation trials 30-60, Late adaptation trials 60-90). Post-blink vergence is the change in vergence across blinks for a given phase, averaged across subjects. Error bars are standard errors of means.

For the decreasing disparity condition, Bonferroni-adjusted pairwise t-test (for six comparisons, $a=0.0085$ ) was not significant when comparing baseline trials with early adaptation trials $(\mathrm{t}(9)=3.302, p=0.036)$, but there was a significant difference in means between baseline trials with late adaptation trials $(t(9)=4.087$, $p=0.004)$. This provides strong evidence for blink adaptation for the decreasing disparity condition. For the increasing disparity condition, the difference in means between baseline trials with early adaptation trials $(\mathrm{t}(9)=-1.156, p=0.731)$ and baseline trials with late adaptation trials $(\mathrm{t}(9)=-2.123, p=0.624)$ was not significant. 


\section{Discussion}

In this experiment, we investigate if a consistent increase or decrease in the retinal disparity of a target across blinks induces blink adaptation. From the results, we infer that over the course of the experiment, the eyes gradually adapt to compensate for the added error in vergence across blinks. This blink adaptation is more effective for errors offset by converging eye movements, as opposed to those offset by diverging eye movements. Furthermore, since the adaptation is in response to a purely binocular cue, we can assume the spatial maps used in error estimation and correction receive consolidated input from both the eyes.

Stereopsis is a computational problem (Marr \& Poggio, 1989). Taking the example of our experiment, first, the brain needs to determine that the two dots presented to each eye separately are not distinct but one and the same. Then, it needs to solve the correspondence problem by spatially matching the dots across the two different retinal images and deriving the disparity. Following this, if the size of the disparity is determined to be outside Panum's fusional area (around $0.17^{\circ}$ (Mitchell, 1966)), an oculomotor signal initiates an appropriate vergence eye movement to ultimately fuse the two retinal images. Additionally, for blink adaptation, a causal association must be established between the blink signal and the vergence error signal. Then for every blink signal, an additional vergence signal is propagated to offset the vergence error.

To solve the aforementioned binocular correspondence problem, it is necessary to combine the visual inputs from the left and right eye. It is only after this that the error in vergence across an eye blink can be detected. On the other hand, if 
we consider the experiments by Maus et al. (2017) and Lau \& Maus (2019), where both eyes receive a conjugate error signal after each blink, the error in gaze targeting can be calculated without combining the inputs from the two eyes.

Thus there is a fundamental difference in the way the error is calculated for blink adaptation processes for conjugate target displacement and blink adaptation for target displacements in depth. Lau \& Maus (2019) showed that even for conjugate displacements of the target, the adaptation gain for both eyes was different, pointing towards a monocular estimation of error and a separate gaze recalibration signal to each eye. The current experiment reveals that blink adaptation can be driven by spatial error maps that combine the visual input from both eyes.

\section{Asymmetry in blink adaptation for vergence}

It is evident from our results that blink adaptation is stronger for the decreasing disparity condition as compared to the increasing disparity condition. Asymmetries were also observed for blink adaptation processes which correct for conjugate errors (Lau \& Maus 2019). Different adaptation gain was found in each eye for target displacements in different directions, with nasal steps leading to better adaptation. Similarly, Albano \& Marrero (1995) and Maiello et al. (2016), found asymmetries in saccadic adaptation with a dichoptic step. They found that the adaptation for a gain decreasing dichoptic step is much stronger compared to a gain increasing adaptation step.

Ideally, error correction in our experiment requires pure vergence eye movements, i.e., to offset the vergence error, a symmetric and disjunctive change in each eye is required. As shown before by Erkelens, Steinman \& Collewijn (1989), 


\section{BLINK ADAPTATION FOR TARGET DISPLACEMENTS IN DEPTH}

however, this type of pure vergence is rarely encountered. In many experiments meant to elicit pure vergence, the vergence movements are accompanied by saccades with the prevalence of saccades higher for tasks requiring divergence movements (Collewijn, Erkelens \& Steinman, 1995). Pure vergence movements are slow. They have a peak velocity on the order of tens of degrees per second as compared to saccades, which reach hundreds of degrees per second (Westheimer, 1954; Rashbass \& Westheimer, 1961). To circumvent this slow response, our eyes sometimes use a combination of vergence and conjugate saccades (Erkelens et al. 1989). During such eye-movements, a saccade directs one eye to fixate on the new target correctly, and the adjacent eye only moves later to correct for the error in vergence. The net result is a diverging/converging eye movement. In the present study, we were not able to determine the nature of the eye movements responsible for blink adaptation, as our video-based eye tracker did not record gaze position during pupil occlusion. We speculate that saccades interfere in vergence adaptation with a higher prevalence of saccades during divergence manifesting in weaker adaptation, as compared to convergence. However, the precise nature of eye movements during blinks is under debate. Blink-related eye movements or 'blink-associated resetting eye movements' (Khazali, Pomper, \& Thier, 2017) are functionally distinct from saccades. It is likely that the eye movements that are adapted during blinks are not saccades, but blink-related eye movements.

Another possible explanation for the contrast in the adaptation for increasing vs. decreasing retinal disparity could be the difference in the dynamics of convergence and divergence eye movements. It has been shown that not only do divergence and convergence eye movements have different velocities and latencies 


\section{BLINK ADAPTATION FOR TARGET DISPLACEMENTS IN DEPTH}

but divergence eye movements, unlike convergence, are also dependent on the initial vergence angle (Alvarez, Semmlow \& Pedrono, 2005; Lee, Chen \& Alvarez, 2008). Furthermore, primate neurophysiological studies show strong evidence for the existence of different cells for convergence and divergence movements (Mays, 1984; Mays et al., 1986). Thus it might be that convergence and divergence are different and independent systems and not merely the inverse of each other. These differences can directly have an effect on the characteristics of adaptation in the two systems.

\section{Conclusion}

The oculomotor system constantly recalibrates our gaze to correct for errors. In this study we build on past literature which demonstrates that conjugate errors induced during blinks results in recalibration of gaze. Here we looked into the question whether our gaze is also adjusted to correct for vergence errors across blinks. Our results suggest that the oculomotor system can adapt to correct for binocular disconjugate vergence errors. Further work may be required to delineate why the adaptation strength for increasing and decreasing disparity conditions are so different.

\section{References}

Albano, J. E., \& Marrero, J. A. (1995). Binocular interactions in rapid saccadic adaptation. Vision Research, 35(23-24), 3439-3450.

doi:10.1016/0042-6989(95)00079-t 
Alvarez, T. L., Semmlow, J. L., \& Pedrono, C. (2005). Divergence eye movements are dependent on initial stimulus position. Vision Research, 45(14), 1847-1855. doi:10.1016/j.visres.2005.01.017

Collewijn, H., Steen, J. V., \& Steinman, R. M. (1985). Human eye movements associated with blinks and prolonged eyelid closure. Journal of Neurophysiology, 54(1), 11-27. doi:10.1152/jn.1985.54.1.11

Collewijn, H., Erkelens, C. J., \& Steinman, R. M. (1995). Voluntary binocular gaze-shifts in the plane of regard: Dynamics of version and vergence. Vision Research, 35(23-24), 3335-3358. doi:10.1016/0042-6989(95)00082-p

Costela, F. M., Otero-Millan, J., Mccamy, M. B., Macknik, S. L., Troncoso, X. G., Jazi, A. N., . . Martinez-Conde, S. (2014). Fixational Eye Movement Correction of Blink-Induced Gaze Position Errors. PLoS ONE, 9(10). doi:10.1371/journal.pone.0110889

Evinger, C. (1995). A Brain Stem Reflex in the Blink of an Eye. Physiology, 10(4), 147-153. doi:10.1152/physiologyonline.1995.10.4.147

Flohr, H., \& Precht, W. (1981). Lesion-induced neuronal plasticity in sensorimotor systems. Springer.

Gamlin, P. D. (2002). Neural Mechanisms for the Control of Vergence Eye Movements. Annals of the New York Academy of Sciences, 956(1), 264-272. doi:10.1111/j.1749-6632.2002.tb02825.x

Green, D. G. (1970). Regional variations in the visual acuity for interference fringes on the retina. The Journal of Physiology, 207(2), 351-356. doi:10.1113/jphysiol.1970.sp009065

Hering, E., Bridgeman, B., \& Stark, L. (1977). The theory of binocular vision. 


\section{BLINK ADAPTATION FOR TARGET DISPLACEMENTS IN DEPTH}

Hopp, J., \& Fuchs, A. F. (2004). The characteristics and neuronal substrate of saccadic eye movement plasticity. Progress in Neurobiology, 72(1), 27-53. doi:10.1016/j.pneurobio.2003.12.002

Iwamoto, Y., \& Kaku, Y. (2010). Saccade adaptation as a model of learning in voluntary movements. Experimental Brain Research, 204(2), 145-162. doi:10.1007/s00221-010-2314-3

Khazali, M. F., Pomper, J. K., \& Thier, P. (2017). Blink associated resetting eye movements (BARMs) are functionally complementary to microsaccades in correcting for fixation errors. Scientific Reports, 7(1). doi:10.1038/s41598-017-17229-w

Kosovicheva, A., \& Bex, P. J. (2020). Perceptual effects of unequal saccadic adaptation produced by a dichoptic step. Journal of Vision, 20(5), 7. doi:10.1167/jov.20.5.7

Lau, W. K., \& Maus, G. W. (2019). Directional biases for blink adaptation in voluntary and reflexive eye blinks. Journal of Vision, 19(3), 13. doi:10.1167/19.3.13

Lee, Y. Y., Chen, T., \& Alvarez, T. L. (2008). Quantitative assessment of divergence eye movements. Journal of Vision, 8(12), 5-5. doi:10.1167/8.12.5

Li, R. W., Tran, K. D., Bui, J. K., Antonucci, M. M., Ngo, C. V., \& Levi, D. M. (2018). Improving Adult Amblyopic Vision with Stereoscopic 3-Dimensional Video Games. Ophthalmology, 125(10), 1660-1662. doi:10.1016/j.ophtha.2018.04.025

Maiello, G., Harrison, W. J., \& Bex, P. J. (2016). Monocular and Binocular Contributions to Oculomotor Plasticity. Scientific Reports, 6(1). doi:10.1038/srep31861 
Marr, D., \& Poggio, T. (1979). A computational theory of human stereo vision. Proceedings of the Royal Society of London. Series B. Biological Sciences, 204(1156), 301-328.

Maus, G. W., Duyck, M., Lisi, M., Collins, T., Whitney, D., \& Cavanagh, P. (2017). Target Displacements during Eye Blinks Trigger Automatic Recalibration of Gaze Direction. Current Biology, 27(3), 445-450.

doi:10.1016/j.cub.2016.12.029

Mays, L. E. (1984). Neural control of vergence eye movements: Convergence and divergence neurons in midbrain. Journal of Neurophysiology, 51(5), 1091-1108. doi:10.1152/jn.1984.51.5.1091

Mays, L. E., Porter, J. D., Gamlin, P. D., \& Tello, C. A. (1986). Neural control of vergence eye movements: Neurons encoding vergence velocity. Journal of Neurophysiology, 56(4), 1007-1021. doi:10.1152/jn.1986.56.4.1007

Mitchell, D. E. (1966). Retinal disparity and diplopia. Vision Research, 6(7-8), 441-451. doi:10.1016/0042-6989(66)90052-6

Pélisson, D., Alahyane, N., Panouillères, M., \& Tilikete, C. (2010). Sensorimotor adaptation of saccadic eye movements. Neuroscience \& Biobehavioral Reviews, 34(8), 1103-1120. doi:10.1016/j.neubiorev.2009.12.010

Savitzky, A., \& Golay, M. J. (1964). Smoothing and Differentiation of Data by Simplified Least Squares Procedures. Analytical Chemistry, 36(8), 1627-1639. doi:10.1021/ac60214a047

A computational theory of human stereo vision. (1979). Proceedings of the Royal Society of London. Series B. Biological Sciences, 204(1156), 301-328. doi:10.1098/rspb.1979.0029 